\title{
INTEGRABLE, ERGODIC ACTIONS OF ABELIAN GROUPS ON VON NEUMANN ALGEBRAS
}

\author{
D. DE SCHREYE
}

\section{Abstract.}

Let $G$ be a fixed locally compact abelian group and $(\mathscr{M}, \alpha)$ a von Neumann algebra with a continuous, faithful, ergodic action of $G$ on $\mathscr{M}$. Let [G] be the set of equivalence classes of covariant isomorphic $G$-systems $(\mathscr{M}, \alpha)$. If $G$ is compact, D. Olesen, G. K. Pedersen, and M. Takesaki proved that there is a product on $[G]$ such that $[G], \times \cong A(\hat{G}, \Pi),$. , the group of anti-symmetric bicharacters of $\hat{G}$. In this paper we prove a similar result for locally compact, non-compact but second countable groups.

\section{Introduction.}

Let $G$ be a locally compact, abelian group, and $(\mathscr{M}, \alpha)$ a von Neumann algebra with a continuous, faithful, ergodic action of $G$ on $\mathscr{M}$. If $G$ is compact, S. Albeverio and R. Høegh-Krohn in [1], and D. Olesen, G. K. Pedersen, and M. Takesaki in [11] showed that the $G$-systems $(\mathscr{M}, \alpha)$, where $G$ is fixed, can be completely classified with the help of $A(\hat{G}, \Pi]$, the anti-symmetric bicharacters of $\hat{G}$.

Since then, a number of attempts to generalize this result have been succesful. A. J. Wassermann in [19] generalized the classification towards nonabelian groups. H. H. Zettl in [20] showed that the ergodicity of $\alpha$ can be weakened down to the condition that the fixed point algebra $\mathscr{M}^{\alpha}$ is contained in the centre of $\mathscr{M}$.

A third generalization which seems natural in this context, is to replace the compactness of $G$ by locally compactness. However, the connection between a $G$-system $(\mathscr{H}, \alpha)$ and a simplectic bicharacter $\chi$ is completely based on the existence of a unitary operator $u_{p}$, for each $p \in \hat{G}$, satisfying $\alpha_{s}\left(u_{p}\right)=\langle s, p\rangle u_{p}$, for all $s \in G$. Of course, this is not an elegant supplementary condition to impose on the $G$-systems. Fortunately we can solve this problem of aesthetics by showing that, under certain separability conditions on $\mathscr{M}$ and $G$, the existence of unitary eigenoperators $u_{p}$ is equivalent to $\alpha$ being an integrable action of $G$. 
This is done in the first paragraph. We also show that in this case the von Neumann algebra has to be semi-finite.

In the second part we prove that, if the same separability conditions are fulfilled and if $\alpha$ is an integrable action of the locally compact abelian group $G$, then the same classification as the one obtained in the compact case will still hold.

I would like to thank my supervisor Alfons Van Daele and my colleagues at the Mathematics Institute of Leuven for their many helpful suggestions.

\section{Integrable actions and unitary eigenoperators.}

Let $G$ be a locally compact group with Haar measure $d s . \mathscr{M}$ is a von Neumann algebra, $\alpha$ a continuous action of $G$ on $\mathscr{M}$. The continuity requirement on $\alpha$ is that each function $s \mapsto \alpha_{s}(x), x \in \mathscr{M}$, is $\sigma$-weakly continuous from $G$ to $\mathscr{M}$. Let $\eta$ be the set of all $x \in \mathscr{M}$ such that there is some $y \in \mathscr{M}$ with $y=\int \alpha_{s}\left(x^{*} x\right) d s$, then $\alpha$ is an integrable action whenever $\mu=$ linear span of $\left\{y^{*} x \mid x, y \in \eta\right\}$ is $\sigma$-weakly dense in $\mathscr{M}$. As a notation we will use

$$
\mathscr{M}_{p}=\left\{\mathrm{x} \in \mathscr{M} \mid \alpha_{s}(x)=\langle s, p\rangle x \text {, for all } s \in G\right\}, \quad p \in \hat{G} .
$$

In order to show that an integrable, ergodic and faithful action admits unitary eigenoperators, an important problem is that it is not clear whether or not for each $p \in \hat{G}$ there is some $x \in \mu$ with $\int \alpha_{s}(x)\langle s, p\rangle^{-1} d s \neq 0$. If so, one can easily verify that

$$
u_{p}=\left\|\int \alpha_{s}(x)\langle s, p\rangle^{-1} d s\right\|^{-1} \cdot \int \alpha_{s}(x)\langle s, p\rangle^{-1} d s
$$

gives us the unitary eigenoperators. We attack the problems as follows.

1.1. LemMA. If $\alpha$ is integrable, then the linear span of $\bigcup_{p \in \hat{G}} \mathscr{M}_{p}$ is $\sigma$-weakly dense in $\mathscr{M}$.

Proof. Let $\varphi$ be in $\mathscr{M}_{*}$ and its restriction to $\mathscr{M}_{p}, \varphi / \mathscr{M}_{p}=0$ for every $p \in \hat{G}$. Take any $x \in \mu$, then $\int \alpha_{s}(x)\langle s, p\rangle^{-1} d s$ is well defined and belongs to $\mathscr{M}_{p}$. This implies

$$
\int \varphi \alpha_{s}(x)\langle s, p\rangle^{-1} d s=0 \quad \text { for every } p \in \hat{G} .
$$

Thus, $\varphi \alpha_{s}(x)=0$ for all $s \in G$ and also $\varphi(x)=0$. Since $\mu$ is dense in $\mathscr{M}, \varphi$ will be 0 . 
1.2. Lemma. If $\alpha$ is ergodic, $K=\left\{p \in \hat{G} \mid \mathscr{M}_{p} \neq\{0\}\right\}$ is a subgroup of $\hat{G}$ and every $\mathscr{M}_{p}, p \in K$, is one-dimensional.

Proof. First observe that if $x \in \mathscr{M}_{p} \backslash\{0\}$, then $x^{*} x$ and $x x^{*}$ are in $\mathscr{M}_{0}$ and by ergodicity of $\alpha$ we have $x^{*} x=\lambda .1$ and $x x^{*}=\mu .1, \lambda, \mu \in \mathbf{R}^{+}$and $|\lambda|=|\mu|$. This means that $x$ is some multiple of a unitary operator. It also shows that $\mathscr{M}_{p}$ is one-dimensional if it is non-zero. If $x$ and $y$ are two operators belonging to the same eigenspace then $\alpha_{s}\left(x^{*} y\right)=x^{*} y$, which shows that $\mathscr{M}_{p}$ is onedimensional if it is non-zero. Now if we have unitaries $u \in \mathscr{M}_{p}$ and $v \in \mathscr{M}_{q}$, then $u v$ is in $\mathscr{M}_{p+q}$ and $u^{*}$ is in $\mathscr{M}_{-p}$, so that $K$ is a group.

1.3. Lemma. If $\alpha$ is a faithful, integrable action, then $K$ is dense in $\hat{G}$.

Proof. Suppose $K$ is not dense. Then there exists an $s \in G \backslash\{e\}$ such that $\langle s, p\rangle=1$ for all $p \in K$. For this $s$ we have $\alpha_{s}(x)=x$ for every $x \in \mathscr{M}_{p}$ and because of Lemma $1.1, \alpha_{s}(x)=x$ for all $x \in \mathscr{M}$. But since $\alpha$ is faithful, this is false.

1.4. Lemma. If $\alpha$ is integrable, then $K$ is open in $\hat{G}$.

Proof. Let $x \in \mu_{+}, \varphi \in\left(\mathscr{M}_{*}\right)_{+}$, and $\varphi(x) \neq 0$. Put $f(s)=\varphi \alpha_{s}(x)$, then $f$ is in $L^{1}(G)$ and

$$
\hat{f}(0)=\int \varphi \alpha_{s}(x) d s>0 .
$$

Since $\hat{f}$ is continuous there is some open part $V$ of $\hat{G}$ containing 0 such that $\hat{f}(p) \neq 0$ for $p \in V$. Hence we have

$$
\int \varphi \alpha_{s}(x)\langle s, p\rangle^{-1} d s \neq 0 \text { and } \int \alpha_{s}(x)\langle s, p\rangle^{-1} d s \neq 0 \quad \text { for all } p \in V .
$$

Because $\int \alpha_{s}(x)\langle s, p\rangle^{-1} d s$ is in $\mathscr{M}_{p}, K$ must be open.

1.5. Lemma. If $\alpha$ is an integrable, ergodic and faithful action, then for every $p \in \hat{G}$ there exists a unitary operator $u$ in $\mathscr{M}$, such that $\alpha_{s}(u)=\langle s, p\rangle u, s \in G$.

Proof. From Lemma 1.2 we have that $K$ is a subgroup of $\hat{G}$. By Lemma 1.4 it must be open, hence it is also closed. Lemma 1.3 shows that $K$ is dense in $\hat{G}$, so that $K=\hat{G}$. This means $\mathscr{M}_{p} \neq\{0\}$ for all $p \in \hat{G}$ and as in Lemma 1.2, we can find unitary operators in each $\mathscr{M}_{p}$.

Next we show that the correspondence between integrability and the existence of unitary eigenoperators works in the other direction as well, if we 
impose separability contions on $\mathscr{M}$ and $G$. So, let us assume that $\forall p \in \hat{G}$, $\exists u \in U(\mathscr{M}), \alpha_{s}(u)=\langle s, p\rangle u$ for all $s \in G$, where $\alpha$ is a continuous, ergodic action of $G$ on $\mathscr{M}$. The subgroup of $U(\mathscr{M})$ consisting of all such eigenoperators will be denoted $G_{\alpha}$. We then have a short exact sequence:

$$
\{1\} \rightarrow \Pi \rightarrow G_{\alpha} \stackrel{\pi}{\rightarrow} \hat{G} \rightarrow\{0\},
$$

where $\pi: u \in G_{\alpha} \mapsto \pi(u) \in \hat{G}$ with $\alpha_{s}(u)=\langle s, \pi(u)\rangle u$ for all $s \in G$. $G_{\alpha}$ is obviously an extension of $\Pi$ by $\hat{G}$, but we have more:

1.6. LемMA. $\pi: G_{\alpha} \rightarrow \hat{G}$ is a continuous map.

Proof. Let $q$ be fixed in $\hat{G}, K$ a compact set of $G$ and $\varepsilon<\frac{1}{2}$. Then take an open set of $\hat{G}$,

$$
O=\{p \in \hat{G}|\langle s, p\rangle-\langle s, q\rangle|\langle\varepsilon \text { for all } s \in K\} .
$$

Assume $u \in \pi^{-1}(O)$. We will construct an open neighbourhood of $u$ contained in $\pi^{-1}(O)$. To do this, observe that $s \mapsto|\langle s, \pi(u)\rangle-\langle s, q\rangle|$ is a continuous function $G \rightarrow R^{+}$. Thus, on a compact set it attains its supremum. Put

$$
\delta=\sup _{s \in K}|\langle s, \pi(u)\rangle-\langle s, q\rangle|<\varepsilon
$$

Next pick any $\varphi \in \mathscr{M}_{*}$ with $|\varphi(u)|=1$, then $s \mapsto \varphi \alpha_{s}(\cdot)$ is a norm-continuous function $G \rightarrow \mathscr{M}_{*}[6]$. Therefore we can find $s_{1}, s_{2}, \ldots, s_{n} \in G$ and open sets

$$
O_{s_{i}}=\left\{s \in G\left|\sup _{\|x\| \leqq 1}\right| \varphi \alpha_{s}(x)-\varphi \alpha_{s_{i}}(x) \mid<\frac{\varepsilon-\delta}{6}\right\}
$$

covering $K$. For the open neighbourhood of $u$ we now choose

$$
O_{u}=\left\{v \in G_{\alpha}|| \varphi \alpha_{s_{1}}(u-v) \mid<\frac{\varepsilon-\delta}{6}, i=0,1, \ldots, n \text { where } s_{0}=0\right\} .
$$

Combining $|\varphi(u)-\varphi(v)|<(\varepsilon-\delta) / 6 \quad$ with $\quad\left|\left\langle s_{i}, \pi(u)\right\rangle \varphi(u)-\left\langle s_{i}, \pi(v)\right\rangle \varphi(v)\right|<$ $(\varepsilon-\delta) / 6$, we obtain that

$$
\left\langle s_{i}, \pi(u)\right\rangle-\left\langle s_{i}, \pi(v)\right\rangle \mid<\frac{\varepsilon-\delta}{3}, \quad i=1,2, \ldots, n \text { and } v \in O_{u} .
$$

On the other hand, for every $s \in K$ there is some $s_{i}$ with $\left|\varphi \alpha_{s}(v)-\varphi \alpha_{s_{i}}(v)\right|<$ $(\varepsilon-\delta) / 6$ for all $v \in O_{u}$ and since

$$
|\varphi(u-v)| \leqq \frac{\varepsilon-\delta}{6}<\frac{1}{2},
$$

we have $|\varphi(v)|>\frac{1}{2}$ so that 


$$
\left|\left\langle s-s_{i}, \pi(v)\right\rangle\right|<\frac{\varepsilon-\delta}{3} .
$$

From the combination of (1), (2), and (3) we then immediately get $O_{u} \in \pi^{-1}(O)$.

Another result concerning $G$ is the following.

1.7. Lemma. If $\mathscr{M}$ acts on a separable Hilbertspace $\mathscr{H}$ and $G$ is second countable, then $G_{\alpha}$ is a Polish space.

Proof. Because $\mathscr{H}$ is separable, $\mathscr{B}(\mathscr{H})_{1}$ is separable and metrisable and so is $G_{\alpha}$. It remains to show that $G_{\alpha}$ is complete. We will verify the completeness in the strong topology. First observe that $\hat{G}$ is a Polish space, since it is second countable, locally compact and Hausdorff [12]. Therefore it is also metrisable and complete. Now, by Lemma 1.6 , if $\left\{u_{i}\right\}$ is a net in $G_{\alpha}$ converging strongly to $x$ in $\mathscr{M}$, then $\left\{\pi\left(u_{i}\right)\right\}$ is a Cauchy net in $\hat{G}$ converging to $p \in \hat{G}$. So, for any fixed $s \in G$ and $\xi \in \mathscr{H}_{1}$ :

$$
\begin{aligned}
&\left\|\left(\alpha_{s}(x)-\langle s, p\rangle x\right) \xi\right\| \leqq\left\|\left(\alpha_{s}(x)-\alpha_{s}\left(u_{i}\right)\right) \xi\right\|+ \\
&+\left\|\left(\left\langle s, \pi\left(u_{i}\right)\right\rangle u_{i}-\langle s, p\rangle u_{i}\right) \xi\right\|+\left\|\left(\langle s, p\rangle u_{i}-\langle s, p\rangle x\right) \xi\right\|,
\end{aligned}
$$

and therefore it must be zero. But then $\alpha_{s}\left(x^{*} x\right)=x^{*} x$, so that by ergodicity of $\alpha, x x^{*}=\lambda .1=x^{*} x$, where $\lambda \in R^{+}$. Furthermore $\|x \xi\|=\lim \left\|u_{i} \xi\right\|=\|\xi\|$ for every $\xi \in \mathscr{H}$, so that $\|x\|=1$. Thus $\lambda=1$ and $x \in G_{\alpha}$.

Probably the conditions we imposed on $\mathscr{M}$ and $G$ are a little too strong for this result, but they will prove easy to handle later on. There is a strong relation between Polish spaces and the existence of a Borel-measurable section for a given function between those spaces. The reason why we brought up the notion of a Polish space is that we want to find such a section for $\pi$.

1.8. Lemma. If $\mathscr{M}$ acts on a separable Hilbert space $\mathscr{H}$ and $\hat{G}$ is second countable, then there exists a Borel map $u: \hat{G} \rightarrow G_{\alpha}$ such that $\pi_{0} u=1_{\hat{G}}$.

Proof. Because of the results in Lemmas 1.6 and 1.7, the conditions of [17, Theorem A.16] are seen to be fulfilled.

In order to have a better view on the situation we recall that if we have a topological group extension $G_{\alpha}$ of $\Pi$ by $\hat{G}$, then there always exists a Borel section $u$ because $\Pi$ is separable [5], [9], [13]. In our short exact sequence 


$$
\{1\} \rightarrow \Pi \rightarrow G_{\alpha} \stackrel{\pi}{\longrightarrow} \hat{G} \rightarrow\{0\}
$$

we meet more difficulties, since $\pi$ is not necessarily open. However, by the separability conditions we imposed, $G_{\alpha}$ is turned into a separable locally compact group and therefore $\pi$ must be open by the Open Mapping Theorem [10].

Later on we will give an example showing that those conditions are not merely due to our lasyness. Without the separability the action need not be integrable. We now have the tools necessary to attack the problem of integrability of $\alpha$.

1.9. LemMA. If $\alpha$ is an ergodic action of $G$ on $\mathscr{M}$, then $\eta=\{0\}$ or $\eta$ is $\sigma$-weakly dense in $\mathscr{M}$.

Proof. $\eta$ is a left ideal of $\mathscr{M}$, so taking the $\sigma$-weak completion $\bar{\eta}$, we get a $\sigma$ weakly closed left ideal with identity $e$ and $\bar{\eta}=\mathscr{M} e$.

Since $\alpha_{t}(\eta) \subset \eta$ and $\alpha_{t}$ is continuous, we have that $\alpha_{t}(\bar{\eta}) \subset \bar{\eta}$ for all $t \in G$. From this $\alpha_{t}(\bar{\eta})=\bar{\eta}$ follows, which in its turn implies $\alpha_{t}(e)=e$ for all $t$. But now, by ergodicity of $\alpha, e=0$ or $e=1$ so that $\eta=0$ or $\eta$ is dense in $\mathscr{M}$.

Because of this Lemma, it will be sufficient to find one integrable, non-zero element in $\mathscr{M}$.

1.10. Lemma. Let $\mathscr{M}$ be a von Neumann algebra acting on a separable Hilbert space $\mathscr{H}$. Denote

$$
y_{f}=\int f(p) u_{p} d p
$$

where $f \in L^{1}(\hat{G}) \cap L^{2}(\hat{G})$ and $p \mapsto u_{p}$ is a section for $\pi$, Borel measurable on the support of $f$, then $y_{f} \in \eta$ and

$$
\int \alpha_{s}\left(y_{f}^{*} y_{f}\right) d s=\|f\|_{L_{2}}^{2} .
$$

Proof. Let $\xi$ be in $\mathscr{H},\|\xi\|=1$, and let $\left\{e_{i}\right\}$ be an orthonormal basis for $\mathscr{H}$, then:

$$
\begin{aligned}
& \int\left\langle\alpha_{s}\left(y_{f}^{*} y_{f}\right) \xi, \xi\right\rangle d s \\
& =\int \sum_{i=1}^{\infty}\left|\left\langle\alpha_{s}\left(y_{f}\right) \xi, e_{i}\right\rangle\right|^{2} d s
\end{aligned}
$$




$$
\begin{aligned}
& =\int \sum_{i=1}^{\infty}\left|\int f(p)\langle s, p\rangle\left\langle u_{p} \xi, e_{i}\right\rangle d p\right|^{2} d s \\
& =\int \sum_{i=1}^{\infty}\left|\mathscr{F}\left(f(\cdot)\left\langle u . \xi, e_{i}\right\rangle\right)(s)\right|^{2} d s .
\end{aligned}
$$

Now apply the monotone convergence theorem and the fact that $\mathscr{F}$ is an isomorphism on $L^{2}(\hat{G})$ to obtain

$$
=\sum_{i=1}^{\infty} \int\left|f(p)\left\langle u_{p} \xi, e_{i}\right\rangle\right|^{2} d p .
$$

Making use of the monotone convergence theorem again, we get

$$
\begin{aligned}
& =\int|f(p)|^{2}\left(\sum_{i=1}^{\infty}\left|\left\langle u_{p} \xi, e_{i}\right\rangle\right|^{2}\right) d p \\
& =\int|f(p)|^{2}\left\|u_{p} \xi\right\|^{2} d p \\
& =\int|f(p)|^{2} d p .
\end{aligned}
$$

In order to obtain the equality for all $\varphi \in \mathscr{M}_{*}$ use the polarisation identity and the fact that we are working on a bounded set in $\mathscr{M}$.

The relation $\int \alpha_{s}\left(y_{f}^{*} y_{f}\right) d s=\|f\|_{L^{2}}^{2}$ should not surprise us. As we will see later on $\mathscr{M} \cong C \times{ }_{m} \hat{G}$, the twisted crossproduct of $C$ by $\hat{G}$ with the 2-cocycle $m(p, q)$ $=u_{p} u_{q} u_{p+q}^{*}$. In this case the Plancherel formula between dual weights (here 1 on $C$ and $\int \alpha_{s}(\cdot) d s$ on $\left.\mathscr{M}\right)$ is known to hold [14], [15].

Combining the results of the Lemmas 1.8, 1.9, and 1.10, the converse of Lemma 1.5 is proved.

1.11. Lemma. If $\mathscr{M}$ is a von Neumann algebra acting on a separable Hilbert space $\mathscr{H}, G$ is a locally compact abelian group with second countable dual $G$, if $\alpha$ is an ergodic, continuous action of $G$ on $\mathscr{M}$, such that

$$
\forall p \in \hat{G}, \exists u \in \dot{U}(\mathscr{M}): \alpha_{s}(u)=\langle s, p\rangle u \quad \text { for all } s \in G,
$$

then $\alpha$ is integrable.

A different proof for Lemma 1.11 can be given using Theorems III 2.12 and III 3.1 from [4]. Such a proof would make use of the notions of dominant- and square integrable $\alpha$-twisted ${ }^{*}$-representations of $G$ in $\mathscr{M}$. The advantage of the proof we gave is that it gives a better view of where the different elements fit in. Unfortunately everything does not work out so nicely when we drop the 
separability condition on $\mathscr{M}$.

1.12. EXAMPLE. Let $G$ be any locally compact, non-compact abelian group and take its Bohr compactification $G_{b}$. Then there exists a continuous isomorphism $\beta: G \rightarrow G_{b}$ such that $\beta(G)$ is a dense subgroup of $G_{b}$ and $\langle p, \beta(s)\rangle$ $=\langle s, p\rangle$ for all $p \in \hat{G}$ and $s \in G$. Now take $\mathscr{M}=L^{\infty}\left(G_{b}\right)$ and for $\alpha: G \rightarrow$ Aut $\mathscr{M}$ the translation $\left(\alpha_{s} f\right)(t)=f(t-\beta(s))$, where $f \in L^{\infty}\left(G_{b}\right)$ and $t \in G_{b}$. This action is obviously continuous and faithful. Moreover, since $G_{b}$ is a compact group, the translation $\left(\tilde{\alpha}_{s} f\right)(t)=f(t-s)$ as an action of $G_{b}$ is ergodic. So, if we take $x \in L^{\infty}\left(G_{b}\right)$ satisfying $\alpha_{s}(x)=x$ for all $s \in G$, then $\tilde{\alpha}_{\beta(s)}(x)=x$ for all $\beta(s) \in \beta(G)$ and using the continuity and ergodicity of $\tilde{\alpha}, x$ must be in $C$.1. Furthermore the action $\tilde{\alpha}$ of $G_{b}$ admits unitary eigenoperators $u_{p}, p \in \hat{G}$, as it was shown in [11]. These operators also satisfy

$$
\alpha_{s}\left(u_{p}\right)=\tilde{\alpha}_{\beta(s)}(u p)=\langle p, \beta(s)\rangle u_{p}=\langle s, p\rangle u_{p},
$$

so that $\left(L^{\infty}\left(G_{b}\right), \alpha\right)$ admits unitary eigenoperators as well. Now, are the separability conditions fulfilled? Well, since we have the property that a locally compact group can only be countable if its topology is discrete [10], $\hat{G}$ must be non-countable. Therefore $\hat{G}$ with the discrete topology on it is a non-second countable group and this in turn implies that $G_{b}$ is non-second countable, because the smallest cardinal number $\alpha$ of a basis for the topology is the same for a group and its dual group [7]. Furthermore, the dimension of $L^{2}(G)$ is always equal to that same cardinal number $\alpha$ associated to the group $G$ [7], thus $L^{2}\left(G_{b}\right)$ is a non-separable Hilbert space.

Finally we come to the key question whether the action is integrable. Well, assume that it is and take any $x \in \eta$, then

$$
\int_{G_{b}} \alpha_{s}\left(\int_{G} \alpha_{t}\left(x^{*} x\right) d t\right) d s \in \text { C. } 1 .
$$

Now take $G$ such that Haar measure on it is $\sigma$-finite (this is certainly the case if $G$ is separable). We can then apply Fubini's Theorem, so that the integral is equal to

$$
\int_{G} \int_{G_{b}} \tilde{\sigma}_{s+\beta(t)}\left(x^{*} x\right) d s d t
$$

By invariance of Haar measure on $G_{b}$ we get $\int_{G} \lambda .1 d t$ which is not defined. So, $\alpha$ cannot be integrable.

Next we show that the semi-finite, normal, faithful, $G$-invariant weight $\varrho$ on $\mathscr{M}$, defined by 


$$
\varrho(x)=\int \alpha_{s}(x) d s,
$$

is a trace on $\mathscr{M}$ if we work within the complete setting of Lemma 1.11 .

To do this we will make use of the standard representation $\left(\pi_{\varrho}, \mathscr{H}_{\varrho}\right)$ of $\mathscr{M}$ associated to $\varrho$. The Hilbert space $\mathscr{H}_{\varrho}$ is obtained from $\mathscr{M}$ and $\varrho$ by taking the pre-Hilbert space $\{\xi(x) \mid x \in \eta\}$ with the inner product $\langle\xi(x), \xi(y)\rangle=\varrho\left(y^{*} x\right)$ and completing it for the norm arising from this inner product. The representation $\pi_{e}$ of $\mathscr{M}$ on $\mathscr{H}_{e}$ is the representation induced by $\pi_{e}(x) \xi(y)$ $=\xi(x y)$. This gives a ${ }^{*}$-isomorphism of $\mathscr{M}$ onto the left von Neumann algebra associated to the left Hilbert algebra $\eta \cap \eta^{*}$ [2], [16]. For the proof that $\varrho$ is a trace, we will need a section $p \mapsto u_{p}$ which is more than just measurable.

1.13. Lemma. Let $\alpha$ be a continuous, integrable and ergodic action of $G$ on $\mathscr{M}$, then there exists a locally continuous section $u: p \in \hat{G} \mapsto u_{p} \in \hat{G}_{\alpha}$. If in addition $G$ is second countable and $\mathscr{M}$ acts on a separable Hilbert space, then $u$ can be chosen a Borel section as well.

Proof. As in Lemma 1.4, let $x$ be in $\mu_{+}$and take any open set $V$ of $\hat{G}$ containing 0 such that

$$
\int \alpha_{s}(x)\langle s, p\rangle^{-1} d s \neq 0 \quad \text { for all } p \in V .
$$

On $V$ we define the section

$$
u: p \in V \mapsto u_{p}=\int \alpha_{s}(x)\langle s, p\rangle^{-1} d s\left\|\int \alpha_{s}(x)\langle s, p\rangle^{-1} d s\right\|^{-1} .
$$

Since the Fourier transform of a function $s \mapsto \varphi \alpha_{s}(x), \varphi \in \mathscr{M}_{*}$, is continuous, $p \mapsto \int \alpha_{s}(x)\langle s, p\rangle^{-1} d s$ is continuous on $V$.

Moreover, $\forall \xi, \eta \in \mathscr{H}$ :

$$
\begin{aligned}
& \left|\left\langle\int \alpha_{s}(x)\langle s, p\rangle^{-1} d s \xi, \eta\right\rangle\right| \\
& \quad=\left|\int\langle s, p\rangle^{-1}\left\langle\alpha_{s}\left(x^{\frac{1}{2}}\right) \xi, \alpha_{s}\left(x^{\frac{1}{2}}\right) \eta\right\rangle d s\right| \\
& \quad \leqq \int\left\|\alpha_{s}\left(x^{\frac{1}{2}}\right) \xi\right\| \cdot\left\|\alpha_{s}\left(x^{\frac{1}{2}}\right) \eta\right\| d s \\
& \quad \leqq\left(\int\left\|\alpha_{s}\left(x^{\frac{1}{2}}\right) \xi\right\|^{2} d s\right)^{\frac{1}{2}}\left(\int\left\|\alpha_{s}\left(x^{\frac{1}{2}}\right) \eta\right\|^{2} d s\right)^{\frac{1}{2}}
\end{aligned}
$$




$$
\begin{aligned}
& =\left(\int\left\langle\alpha_{s}(x) \xi, \xi\right\rangle d s\right)^{\frac{1}{2}}\left(\int\left\langle\alpha_{s}(x) \eta, \eta\right\rangle d s\right)^{\frac{1}{2}} \\
& \leqq\left\|\int \alpha_{s}(x) d s\right\| \cdot\|\xi\| \cdot\|\eta\| .
\end{aligned}
$$

So that $\left\|\int \alpha_{s}(x)\langle s, p\rangle^{-1} d s\right\| \leqq\left\|\int \alpha_{s}(x) d s\right\|$ and since the norm-function is $\sigma$ weakly lower continuous, $p \mapsto u_{p}$ is continuous on $V$.

Next we extend this section to the whole of $\hat{G}$. To do this, observe that $V^{c}$ and $\left(\pi^{-1}(V)\right)^{c}$ are both closed and therefore Polish subspaces of $\hat{G}$ and $G_{\alpha}$. Also, the restriction of $\pi$ stays continuous from $\left(\pi^{-1}(V)\right)^{c}$ onto $V^{c}$. Therefore, the conditions of [17, Theorem A.16] are fulfilled again and we have a Borel section $u^{\prime}: V^{c} \rightarrow G_{\alpha}$. Finally, take the section $u+u^{\prime}: \hat{G} \rightarrow G_{\alpha}$.

Now, using the section $u+u^{\prime}$, we see that the Hilbert space $\mathscr{H}_{p}$ appears to be rather elegant. We work with the conditions of Lemma 1.11.

\subsection{LemMa. $\mathscr{H}_{e} \cong L^{2}(\hat{G})$.}

Proof. Let $\mathscr{A}$ be the set $\left\{y_{f} \mid f \in L^{1}(\hat{G}) \cap L^{2}(\hat{G})\right\}$ where we use the locally continuous Borel section of Lemma 1.13 in the integral for $y_{f}$. First we show that $\mathscr{A}$ is $\sigma$-weakly dense in $\mathscr{M}$.

Indeed, let $\varphi \in \mathscr{M}_{*}$ and $\varphi\left(y_{f}\right)=0$ for all $y_{f}$ in $\mathscr{A}$. Then $\varphi\left(u_{p}\right)=0$ almost everywhere. Suppose that $\varphi\left(u_{q}\right) \neq 0$ for some $q \in \hat{G}$, then $p+q \mapsto v_{p+q}=u_{q} u_{p}$ is a new Borel section for $\pi$ which is continuous at $q$. For this section as well we have $\varphi\left(v_{p}\right)=0$ almost everywhere and $\varphi\left(v_{q}\right) \neq 0$ since $v_{r}=\lambda_{r} u_{r}$ for all $r \in \hat{G}$, $\lambda_{r} \in \Pi$. But this contradicts the continuity of $v$ at $q$, so that $\varphi\left(u_{p}\right)=0$ for all $p \in \hat{G}$. On its turn this implies $\varphi=0$, because $\left\{u_{p} \mid p \in \hat{G}\right\}$ has a dense linear span in $\mathscr{M}$ by Lemma 1.1 .

$\mathscr{A}$ has some fine properties. First, from Lemma 1.10 we see that

$$
\int \alpha_{s}\left(y_{f}^{*} y_{g}\right) d s=\langle g, f\rangle_{L^{2}}
$$

and a similar proof as the one of Lemma 1.10 gives us

$$
\int \alpha_{s}\left(y_{\mathrm{g}} y_{f}^{*}\right) d s=\langle\mathrm{g}, f\rangle_{L^{2}}
$$

as well. So, $\varrho$ is a trace on $\mathscr{A}$. A second property is that $\mathscr{A}$ is closed for the involution. We have

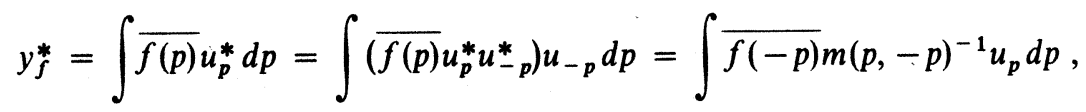


where $m$ is the 2-cocycle in $Z^{2}(\hat{G}, \Pi)$ defined by $m(p, q)=u_{p} u_{q} u_{p+q}^{*}$. Since $m$ is bounded and Borel measurable, the function

$$
p \mapsto \overline{f(-p)} \cdot m(p,-p)^{-1}
$$

is in $L^{1}(\hat{G}) \cap L^{2}(\hat{G})$ again.

Next we take a look at the pre-Hilbert space $\xi(\mathscr{A}) \subset \mathscr{H}_{Q}$. Taking its norm completion we obtain a sub-Hilbert space of $\mathscr{H}_{e}$ which is isomorphic to $L^{2}(\hat{G})$ as was demonstrated in Lemma 1.10 .

Now let $x$ be a linear combination from the $u_{p}$ 's and $\xi\left(y_{f}\right) \in \xi(\mathscr{A})$, then $\pi_{\boldsymbol{Q}}(x) \xi\left(y_{f}\right) \in \xi(\mathscr{A})$. Furthermore, if $x \in \mathscr{M}$, then there is a net $\left\{x_{i}\right\}$ of linear combinations of the $u_{p}$ 's and $x_{i} \rightarrow x \sigma$-weakly. By $\sigma$-weak continuity of $\pi$, $\pi\left(x_{i}\right) \rightarrow \pi(x)$ and in particular $\pi\left(x_{i}\right) \xi\left(y_{f}\right) \rightarrow \pi(x) \xi\left(y_{f}\right)$ in $\mathscr{H}_{e}$ and therefore also in $L^{2}(\hat{G})$. So, $\xi\left(x y_{f}\right) \in L^{2}(\hat{G})$ for each $x \in \mathscr{M}$ and $y_{f} \in \mathscr{A}$.

We next investigate multiplication from the right. Therefore let $x \in \eta$ and $y_{f} \in \mathscr{A}$, then

$$
\xi\left(y_{f} \cdot x\right)=\xi_{\left(x^{*}, y_{j}^{*}\right)^{*}}
$$

will be in $L^{2}(\hat{G})$ again if we can prove that for every $z \in \eta \cap \eta^{*}, \xi(z) \in L^{2}(\hat{G})$ implies $\xi_{\left(z^{*}\right)} \in L^{2}(\hat{G})$. To prove this, let $\left\{z_{n}\right\}$ be a net in $\mathscr{A}$ such that $\xi_{\left(z_{n}\right)} \rightarrow \xi_{(z)}$, then

$$
\left\|\xi_{\left(z_{n}^{*}\right)}-\xi_{\left(z_{m}^{*}\right)}\right\|^{2}=\int \alpha_{s}\left(z_{n}-z_{m}\right)\left(z_{n}-z_{m}\right) * d s=\left\|\xi_{\left(z_{n}\right)}-\xi_{\left(z_{m}\right)}\right\|^{2}
$$

since $\varrho$ is a trace on $\mathscr{A}$. Thus, $\left\{\xi_{\left(z_{n}^{*}\right)}\right\}$ also converges. Now there is a densely defined linear operator $S$ on $\mathscr{H}_{e}$,

$$
S: \xi_{(z)} \in \xi(\eta) \mapsto \xi_{\left(z^{*}\right)},
$$

which is pre-closed [2]. By this property of $S$, the facts that $\left\{\xi_{\left(z_{n}^{*}\right)}-\xi_{\left(z^{*}\right)}\right\}$ converges and $\xi_{(z)_{n}}-\xi_{(z)} \rightarrow 0$ imply that $\xi_{\left(z_{n}^{*}\right)}-\xi_{\left(z^{*}\right)} \rightarrow 0$. Hence $\xi_{z^{*}} \in L^{2}(\hat{G})$ and therefore $\xi\left(y_{f} \cdot x\right) \in L^{2}(\hat{G})$.

Finally we use the $\sigma$-weak continuity of $\pi_{e}$ again and the fact that $\mathscr{A}$ is $\sigma$ weakly dense in $\mathscr{M}$ to find that $\xi_{(x y)} \in L^{2}(\hat{G})$ for all $x \in \mathscr{M}, y \in \eta$ and so $\xi(\eta)$ $\subset L^{2}(\hat{G})$ and $L^{2}(\hat{G}) \cong \mathscr{H}_{Q}$.

With this Lemma, all "hard labour" has been done in our quest to find the Holy Trace.

1.15. Proposition. If $\mathscr{M}$ is a von Neumann algebra acting on a separable Hilbert space $\mathscr{H}$ and $G$ is a locally compact, second countable, abelian group, if $\alpha$ is an ergodic, continuous and integrable action of $G$ on $\mathscr{M}$, then $\varrho(x) .1=\int \alpha_{s}(x) d s$ defines a $G$-invariant, normal, semi-finite faithful trace on $\mathscr{M}$. 
Proof. In the proof of Lemma 1.13 we found that $\varrho$ is a trace on a dense part $\mathscr{A}$. We also saw that if $z \in \eta \cap \eta^{*}$ and if $\left\{z_{n}\right\}$ is a net in $\mathscr{A}, \xi\left(z_{n}\right) \rightarrow \xi(z)$, then $\xi_{\left(z_{n}^{*}\right)} \rightarrow \xi_{\left(z^{*}\right)}$ By the conclusion of Lemma 1.13 such a net always exists. Thus for any $z \in \eta \cap \eta^{*}$ :

$$
\begin{aligned}
\int \alpha_{s}\left(z^{*} z\right) d s & =\|\xi(z)\|^{2}=\lim \left\|\xi\left(z_{n}^{*}\right)\right\|^{2}=\lim \left\|\xi\left(z_{n}^{*}\right)\right\|^{2} \\
& =\left\|\xi\left(z^{*}\right)\right\|=\int \alpha_{s}\left(z z^{*}\right) d s
\end{aligned}
$$

An immediate consequence is that for all

$$
x, y \in \eta \cap \eta^{*}: \int \alpha_{s}\left(x y^{*}\right) d s=\int \alpha_{s}\left(y^{*} x\right) d s .
$$

Now from Proposition 3.8 of [3] we may conclude that the restriction of $\varrho$ to the von Neumann algebra obtained in the $\sigma$-weak completion of $\eta \cap \eta^{*}$ is a trace. But since $\mu \subset \eta \cap \eta^{*}$ and $\alpha$ is integrable, this von Neumann algebra is $\mathscr{M}$.

\section{Integrable $G$-systems.}

2.1. From now on let $G$ be a fixed locally compact, second countable abelian group. A pair $(\mathscr{M}, \alpha)$ will be called an integrable $G$-system, whenever $\mathscr{M}$ is a von Neumann algebra acting on a separable Hilbert space and $\alpha$ is a faithful, continuous, ergodic and integrable action of $G$ on $\mathscr{M}$. It is not clear whether the separability conditions are still essential here, but in the sense of Lemma 1.11 they provide us with an easy way of verifying when an action is integrable.

We define the product of 2 integrable $G$-systems as it is done in [11] for $G$ systems. This means: $(\mathscr{M}, \alpha) \times(\mathscr{N}, \beta)=(\mathscr{P}, \alpha \otimes 1)$, where $\mathscr{P}$ is the fixed point algebra of $\mathscr{M} \otimes \mathscr{N}$ under the action $s \rightarrow \alpha_{s} \otimes \beta_{-s}$.

By Lemma 1.5, for each $p \in \hat{G}$, there is a corresponding unitary eigenoperator $u$ in $\mathscr{M}$ and $v$ in $\mathscr{N}$. Then, $u \otimes v$ is a unitary eigenoperator corresponding to $p$ in $\mathscr{P}$.

The verifications on $(\mathscr{P}, \alpha \otimes 1)$ are now easy. Faithfulness and continuity of $\alpha \otimes 1$ are no problem, nor is the separability of the Hilbert space. To see that $\alpha \otimes 1$ is ergodic, suppose that $x \in \mathscr{P}$ and for all $s \in G,\left(\alpha_{s} \otimes 1\right)(x)=x$. Then take any $\varphi \in \mathscr{M}_{*}$ and $\psi \in \mathscr{N}_{*}$ and apply $\varphi \otimes \psi$ to the previous equality. We obtain:

$$
\varphi \alpha_{s}[(1 \otimes \psi)(x)]=\varphi[(1 \otimes \psi)(x)] \quad \text { for all } \varphi \in \mathscr{M}_{*} \text { and } s \in G .
$$

Since $\alpha$ is ergodic, this means that there is some $\lambda_{\psi} \in \mathrm{C}$ so that: $(1 \otimes \psi)(x)$ 
$=\lambda_{\psi} .1$. The same procedure applied to the equality $\left(1 \otimes \beta_{s}\right)(x)=x$ shows that there exists a $\lambda_{\varphi} \in \mathrm{C}$ so that $(\varphi \otimes 1)(x)=\lambda_{\varphi}$. 1. Thus,

$$
\lambda_{\psi} \varphi(1)=(\varphi \otimes \psi)(x)=\lambda_{\varphi} \psi(1) .
$$

Now if $\psi$ is such that $\psi(1) \neq 0$, we get

$$
(\varphi \otimes \psi)(x)=\frac{\lambda_{\psi}}{\psi(1)} \cdot(\varphi \otimes \psi) \quad \text { and } \quad x=\lambda \psi / \psi(1) \cdot 1,
$$

where $\lambda \psi / \psi(1)$ is now independent of $\psi$. So, $\alpha \otimes 1$ is ergodic. Finally, the integrability of $\alpha \otimes 1$ follows from Lemma 1.11 .

2.2. As it was done in [11], we will make use of an equivalence relation on the set of all integrable $G$-systems: $(\mathscr{M}, \alpha) \sim(\mathcal{N}, \beta)$ whenever there exists an isomorphism $\varphi: \mathscr{M} \rightarrow \mathscr{N}$, so that $\varphi_{0} \alpha_{s} \circ \varphi^{-1}=\beta_{s}$, for all $s \in G$. $[\alpha]$ denotes the equivalence class containing $(\mathscr{M}, \alpha)$ and $[G]$ is the set of all equivalence classes. The product of 2 classes is defined as the class of the product of 2 representatives. One can easily check that this is well defined.

2.3. Next we observe that to any integrable $G$-system we have an associated Borel 2-cocycle $m \in Z_{b}^{2}(\hat{G}, \pi)$,

$$
m(p, q)=u(p) u(q) u(p+q)^{*},
$$

where $u: \hat{G} \rightarrow G_{\alpha}$ is a Borel section for $\pi$. Since the linear space $\mathscr{M}_{p}, p \in \hat{G}$, are all 1-dimensional, a different choise for $u$ does not change the equivalence class corresponding to $m$ in

$$
H_{b}^{2}(\hat{G}, \Pi)=Z_{b}^{2}(\hat{G}, \Pi) /_{B_{b}^{2}(\hat{G}, \Pi)} .
$$

As it was done in the compact case, we will classify integrable $G$-systems by means of $H_{b}^{2}(\hat{G}, \Pi)$. However, we would be viciously misleading the reader, if at the same time we did not give him sufficient tools to calculate $H_{b}^{2}(\hat{G}, \Pi)$. To this end, we recall the following result, which is well known in the theory of multipliers. (See for instance [8].)

2.4. Lemma. Let $G$ be a locally compact abelian group, then there exists an isomorphism $\varphi: m \mapsto m \cdot \hat{m}^{-1}$, from $H_{b}^{2}(G, \Pi)$ into $A(G, \Pi)$, the anti-symmetrical bicharacters of $G \times G$. $(\hat{m}(x, y)=m(y, x))$.

Although this result enables us to compute $H_{b}^{2}(\hat{G}, \Pi)$ in many cases, an "onto"-isomorphism instead of "into" would be preferable. Unfortunately, as far as we know it is an unsolved problem whether or not such an isomorphism 
exists for every locally compact abelian group. It was however obtained for every one of the following special cases [8]:

a) $G$ is discrete.

b) $G / K$ is a union of compact open subgroups and the 2-primary component of $G$ is a direct product of a compact group and a discrete group, where $K$ is the connected component of the identity of $G$. (special case: $G$ is compact).

c) $x \mapsto x^{2}$ is an automorphism of $G$.

d) $G$ is a direct product of the above 2 types.

2.5. LemMA. If $m \in Z_{b}^{2}(\hat{G}, \Pi)$, then there exists an integrable $G$-system $\left(\mathscr{M}^{m}, \alpha^{m}\right)$ such that it admits a map:

$$
p \in \hat{G} \mapsto u_{p} \in G_{\alpha} \text { satisfying } m(p, q)=u_{p} u_{q} u_{p+q}^{*} .
$$

Proof. For the von Neumann $\mathscr{M}^{m}$ we take $C \times_{m} \hat{G}$, which is a special case of an $m$-twisted cross product as it was defined in [15]. We recall that this is the sub-von Neumann algebra of $\mathscr{B}\left(L^{2}(\hat{G})\right)$ generated by the operators $u_{p}=\lambda_{p} m_{m(p, .)}$, where

$$
(\lambda p(f))(q)=f(q-p) \quad \text { and } \quad\left(m_{m(p, .)}(f)\right)(q)=m(p, q) f(q) .
$$

From this, we immediately obtain $u_{p} u_{q} u_{p+q}^{*}=m(p, q)$. On this algebra we use the action of $G$ implemented by the left regular representation

$$
v:\left(v_{s}(f)\right)(p)=\langle s, p\rangle f(p) .
$$

Straightforward calculation shows: $\alpha_{s}^{m}\left(u_{p}\right)=\langle s, p\rangle u_{p}$.

Observe that $\alpha^{m}$ is continuous and faithful and that $L^{2}(\hat{G})$ is separable, since $\hat{G}$ is a locally compact, second countable Hausdorff space [12].

The ergodicity of $\alpha^{m}$ can be seen as follows. Assume that $\alpha_{s}^{m}(x)=x$ for some $x \in \mathscr{B}\left(L^{2}(\hat{G})\right)$ and all $x \in G$. Then $x$ commutes with all $v_{s}$ and therefore also with $\left\{v_{s} \mid s \in G\right\}^{\prime \prime}=L^{\infty}(\hat{G})[18, \mathrm{~B} 2]$. Now, with a simple verification one can see that each $u_{p}$ commutes with all $\lambda_{q} m_{m(., q)}$, so that in combination with the result above $x$ commutes with every $\lambda_{q} m_{f}, f \in L^{\infty}(\hat{G})$. But these last operators are dense in $\mathscr{B}\left(L^{2}(\hat{G})\right)$ so that $x \in C .1$ and $\alpha$ is ergodic. Again the integrability of $\alpha^{m}$ is clear from Lemma 1.11 .

Next we show the uniqueness of this system up to equivalence *

2.6. LeMMA. If $m \in Z_{b}^{2}(\hat{G}, \Pi)$ and $(\mathscr{M}, \alpha)$ and $(\mathcal{N}, \beta)$ are integrable $G$-systems both admitting a Borel map $p \in \hat{G} \mapsto u_{p} \in G_{\alpha}$ (or $G_{\beta}$ ) satisfying $m(p, q)$ $=u_{p} u_{q} u_{p+q}^{*}$, then $(\mathscr{M}, \alpha) \sim(\mathcal{N}, \beta)$. 
Proof. We will work in the standard representation of $\mathscr{M}$ associated to $\varrho_{\alpha}$. According to Lemma 1.14 the representation $\pi_{\ell_{a}}$ gives a ${ }^{*}$-isomorphism of $\mathscr{M}$, representing it on $L^{2}(\hat{G})$. If $q \mapsto u_{q}$ is the locally continuous Borel section obtained in Lemma 1.13, then

$$
\pi\left(u_{q}\right) \int f(p) u_{p} d p=\int f(p) m(p \cdot q) u_{p+q} d p
$$

for all $f \in L^{1}(\hat{G}) \cap L^{2}(\hat{G})$.

In the same way $\mathscr{N}$ in represented on $L^{2}(\hat{G})$ by $\pi_{e_{\beta}}$ and if $q \mapsto v_{q}$ is a locally continuous Borel section, we have that

$$
\pi_{Q_{B}}\left(v_{q}\right) \int f(p) v_{p} d p=\int f(p) m(p . q) v_{p+q} d p .
$$

Now the linear span of the $u_{q}, q \in \hat{G}$, is $\sigma$-weakly dense in $\mathscr{M}$ by Lemma 1.1 and so is the span of $v_{q}, q \in \hat{G}$, in $\mathscr{N}$. Moreover, these operators act in the same way on the same Hilbert space. Therefore the ${ }^{*}$-isomorphism $\varphi: u_{q} \rightarrow v_{q}$ between the $2^{*}$-algebras they generate extends to an isomorphism of the enveloping von Neumann algebras. We clearly have: $\varphi_{0} \alpha_{s}=\beta_{s} \circ \varphi$, for all $s \in G$.

Finally we can generalize the isomorphism of $[G], \times$ onto $H^{2}(\hat{G}, \Pi)$ in the locally compact setting.

2.7. ThEOREM. The groups $[G], \times$ and $H_{b}^{2}(\hat{G}, \Pi)$ are isomorphic.

Proof. This is an easy consequence of $2.1,2.3$, and the Lemma 2.5 and 2.6.

\section{REFERENCES}

1. S. Albeverio and R. Høegh-Krohn, Ergodic actions by compact groups on von Neumann algebras, Math. Z. 174 (1980), 1-17.

2. F. Combes, Poids associé à une algèbre Hilbertiènne à gauche, Compositio Math. 23 (1971), 4977.

3. F. Combes, Poids et espérances conditionelles dans les algèbres de von Neumann, Bull. Soc. Math. France 99 (1971), 73-112.

4. A. Connes and M. Takesaki, The flow of weights on a factor of type III, Tohoku Math. J. 29 (1977), 473-573.

5. J. Feldman and F. P. Greenleaf, Existence of Borel transversals in groups, Pacific J. Math. 25 (1968), 455-461.

6. A. Guichardet, Systèmes dynamiques non commutatifs (Astérisque 13-14), Société Mathématique de France, Paris, 1974.

7. E. Hewitt and K. A. Ross, Abstract harmonic an analysis I (Grundlehren Math. Wiss. 115), Springer-Verlag, Berlin - Heidelberg - New York, 1963.

8. A. Kleppner, Multipliers on Abelian Groups, Math. Ann. 158 (1965), 11-34. 
9. G. W. Mackey, Les ensembles Boreliens et les extensions des groupes, J. Math. Pures Appl. 36 (1957), 171-178.

10. S. A. Morris, Pontryagin duality and the structure of locally compact abelian groups (London Math. Soc. Lecture Note Ser. 29), Cambridge Univ. Press, Cambridge, 1977.

11. D. Olesen, G. K. Pedersen, and M. Takesaki, Ergodic actions of compact abelian groups, J. Operator Theory 3 (1980), 237-269.

12. G. K. Pedersen, $\mathrm{C}^{*}$-algebras and their automorphism groups (London Mathematical Society Monographs 14) Academic Press, London, New York, San Francisco, 1979.

13. M. Rieffel, On extensions of locally compact groups, Amer. J. Math. 88 (1966), 871-880.

14. C. E. Sutherland, Cohomology and extensions of von Neumann algebras I, Publ. Res. Inst. Math. Sci. 16 (1980), 105-133.

15. C. E. Sutherland, Cohomology and extensions of von Neumann algebras II, Publ. Res. Inst. Math. Sci. 16 (1980), 135-174.

16. M. Takesaki, Tomita's theory of modular Hilbert algebras and its applications (Lecture Notes in Math. 128), Springer-Verlag, Berlin - Heidelberg - New York, 1970.

17. M. Takesaki, Theory of operator algebras I, Springer-Verlag, Berlin - Heidelberg - New York, 1979.

18. A. Van Daele, Continuous crossed products and type III von Neumann algebras (London Math. Soc. Lecture Note Ser. 31), Cambridge Univ. Press, Cambridge, 1976.

19. A. J. Wassermann, Automorphic actions of compact groups on operator algebras, Thesis, Pennsylvania University, 1981.

20. H. H. Zettle, On compact abelian W*-dynamical systems I, Preprint, Saarbrücken, 1982.

\author{
KATHOLIEKE UNIVERSITEIT LEUVEN \\ DEPT. WISKUNDE \\ CELESTIJNENLAAN $200 \mathrm{~B}$ \\ B-3030 HEVERLEE \\ BELGIUM
}

